\title{
Importance of lotus effect on surface sensing
}

Stacey Sova, Narasimha Prasad, Christopher Cooper, Lisa Kelly, Bradley Arnold, et al.

Stacey Sova, Narasimha Prasad, Christopher Cooper, Lisa Kelly, Bradley Arnold, Brian Cullum, Fow-Sen Choa, N. B. Singh, "Importance of lotus effect on surface sensing," Proc. SPIE 11020, Smart Biomedical and Physiological Sensor Technology XV, 1102005 (2 May 2019); doi: 10.1117/12.2519738

Event: SPIE Defense + Commercial Sensing, 2019, Baltimore, Maryland, United States 


\title{
Importance of Lotus Effect on Surface Sensing
}

\author{
Stacey Sova, Narasimha Prasad*, Christopher Cooper, Lisa Kelly, Bradley Arnold, Brian \\ Cullum, Fow-Sen Choa and N. B. Singh \\ Department of Chemistry and Biochemistry, University of Maryland Baltimore County, 1000 \\ Hilltop Circle, Baltimore, MD, USA 21250 \\ *NASA Langley Research Center, 5 North Dryden Street, Hampton, VA 23681
}

\begin{abstract}
Superhydrophobic polymer films are a material of interest for aircraft deicing fluids to achieve the selfcleaning lotus effect. Hydrophobic polymer films were obtained by a solvent selective method composed of hydrophilic polymethylmethacrylate (PMMA) and hydrophobic polystyrene (PS) and hydrophilic titania nanoparticles. The addition of titania nanoparticles changed the surface of the thin films from an anisotropic morphology to a spherical isotropic surface due to hydrophobic and hydrophilic repulsion. Irradiation of UV responsive titania nanoparticles retained the same surface morphology. Water contact angle measurements will be completed to determine the hydrophobic nature of the polymer films.
\end{abstract}

\section{INTRODUCTION}

The aircraft deicing fluid (ADF) materials have been classified in many categories. The process of deicing for high altitude aircrafts is very complex. The deicing fluids which possess the characteristics of pseudoplatics, generally have some thickening agents to avoid immediate removal of deicing materials by flow process from the surface of the aircrafts. The fluid film stays in place until the aircraft flies with speed in the range $>200 \mathrm{KM} / \mathrm{h}$. In the vicinity of this speed, shear stress causes the breakdown of viscosity. The high speeds required for viscosity breakdown makes this class of fluid very suitable for larger aircraft. To achieve some longer holdover, this class of liquids are modified and dyed green to aid in the application of a consistent layer of fluid. Propylene glycol-based fluids are favored because of less toxicity than ethylene glycol. other ingredients in these materials are thickening agents, wetting agents and UV sensitive dyes. For this purpose, pure benzotriazole (also methyl-substituted benzotriazole) for corrosion inhibitor/flame retardants, Alkylphenol and alkylphenol ethoxylates for nonionic surfactants to reduce surface tension, and triethanolamine for $\mathrm{pH}$ buffer are some chemicals used in commercial materials. In addition, high molecular weight, polymers to increase viscoelasticity and azo, and anthroquinone dyes are used to aid in identification. Ice dendrites along with atmospheric impurities are deposited on the surface of aircrafts by solidification process. The lotus-effect which is a natural phenomenon observed on the surface of the lotus flower leaves and refers to the high-water repellence can be embodied in deicing process. ${ }^{1}$ Nanotechnology provides solutions for selfcleaning, dirt and water-repellent coatings. However, by embodiment of nanoengineered composition (such as potassium titanium oxide/oxalates), there is potential for self-cleaning on grounds followed by use of improved composition of above mentioned chemicals technology can be used for even smaller aircrafts. ${ }^{1}$ On the ground a concept of superhydrophobia can be used, while as slight viscous designed chemicals will help for deicing at high altitudes.

The lotus effect as well as superhydrophobic polymers embody the self-cleaning effect due to the surface structure and composition at the micro and nanoscale level. ${ }^{1}$ The surface hydrophobicity or wettability is a measure of both the surface composition and roughness. ${ }^{1-4}$ Polymers are widely used to generate superhydrophobic polymers, polystyrene (PS) as the most frequently used. There are several methods to prepare these thin films such as the selective solvent method, ethching or electrospinning. ${ }^{1}$ 
In order to achieve roughness in these thin films, solvents remove polymer material and leave a pocket where it previously was generating a rough surface. ${ }^{2}$ Interest in nanoparticles aiding in the generation a rough surface has increased due to their size tunability in the nanometer realm. UV responsive materials such as titania have been used to generate hydrophilic surfaces from irradiation of hydrophobic surfaces. ${ }^{3}$ However, titania nanoparticles are hydrophilic in nature. Combining both the selective solvent method and UV responsive titania nanoparticles, we propose a method to generate hydrophobic thin films from hydrophilic polymethylmethacrylate (PMMA), PS and titania nanoparticles.

\section{EXPERIMENTAL METHODS}

\subsection{Titania nanoparticle synthesis}

Sol-gel synthesis of titania nanoparticles followed procedure previously published with slight modification. ${ }^{5}$ Titanium tetrachloride $(4 \mathrm{~mL})$ was added to $10 \mathrm{~mL} 95 \%$ ethanol over ice and under nitrogen. Aqueous acetic acid (5 $\mathrm{mL} 15 \mathrm{M}$ acetic acid and $40 \mathrm{~mL}$ water) was added to the titanium solution over ice and under a nitrogen flow. The solution was then refluxed with stirring at $50{ }^{\circ} \mathrm{C}$ for 1 hour, and then $60{ }^{\circ} \mathrm{C}$ for another hour until the clear solution became opaque. The resulting nanoparticles were characterized with DLS, absorption and IR measurements. Ground state UV-vis absorption spectra were measured using a JASCO V-570 double beam spectrophotometer. Infrared measurements were obtained with a Thermo Scientific Nicolet IS50 FTIR.

\subsection{Polymer thin film synthesis}

A 70/30 weight percent of polystyrene (PS) and polymethylmethacrylate (PMMA) (1.9 g and $0.9 \mathrm{~g}$ ) were dissolved with heat in $40 \mathrm{~mL}$ THF to form a $5 \%$ weight solution. ${ }^{2}$ Thin films containing nanoparticles added $10 \mathrm{~mL}$ of the titania solution to the PS and PMMA solution. ${ }^{3}$ The polymer films were spincast onto a glass slide by $2000 \mathrm{rpm}$ for 90 seconds at room temperature on a Chemat Technology Spin Caster KW-4A. One nanoparticle sample was layered over the polystyrene and polymethylmethacrylate thin film. The samples were then washed with cyclohexane and heated at $100{ }^{\circ} \mathrm{C}$ for 20 minutes. $^{2}$ Polymers containing nanoparticles were irradiated with a $450 \mathrm{~W}$ mercury vaporlamp (Ace Glass, Vineland, NJ) for 3 hours and kept at constant room temperature.

The surface morphology of the films were characterized by scanning electron microscopy. The chemical composition of the films were characterized with absorption and fluorescence and infrared spectroscopy (FTIR). Images were taken via a FEI NanoSEM Nova-450.

\section{RESULTS AND DISCUSSION}

\subsection{Titania Nanoparticle Characterization}

Titania nanoparticles were synthesized via an aqueous sol-gel method at $\mathrm{pH} 1$. Synthesis yielded an opaque white solution in aqueous media shown in Figure 2. The aqueous titania nanoparticles were characterized by UV-Vis and infrared spectroscopy. The infrared spectrum in Figure 1 displays peaks at $1640 \mathrm{~cm}^{-1}$ attributed to $\mathrm{O}-\mathrm{H}$ stretching, peaks appearing at $1075 \mathrm{~cm}^{-1}$ are attributed to Ti-O-C stretching, and peaks below $700 \mathrm{~cm}^{-1}$ were attributed to Ti-OTi stretching according to literature. ${ }^{5}$ The absorbance of the aqueous nanoparticles had a peak

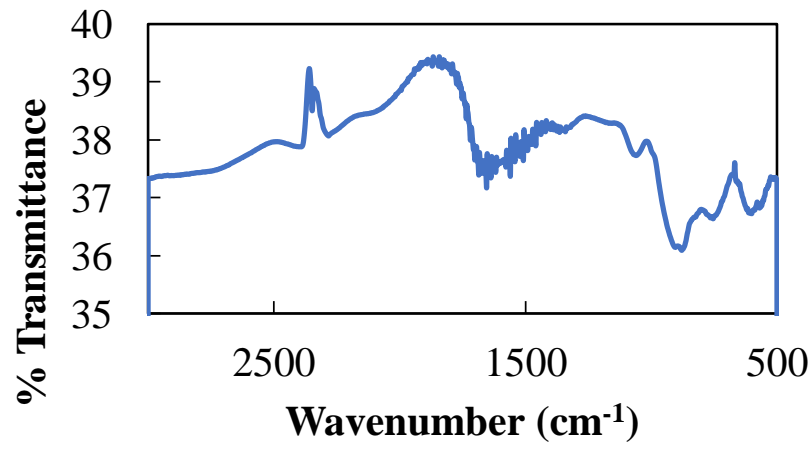

Figure 1. IR spectrum of titania nanoparticles in aqueous solution $\mathrm{pH} 1$.

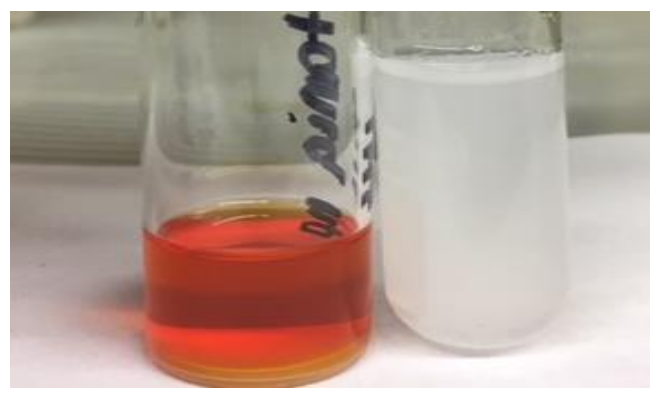

Figure 2. Titania nanoparticles in THF (left) and aqueous solution $\mathrm{pH} 1$ (right). 


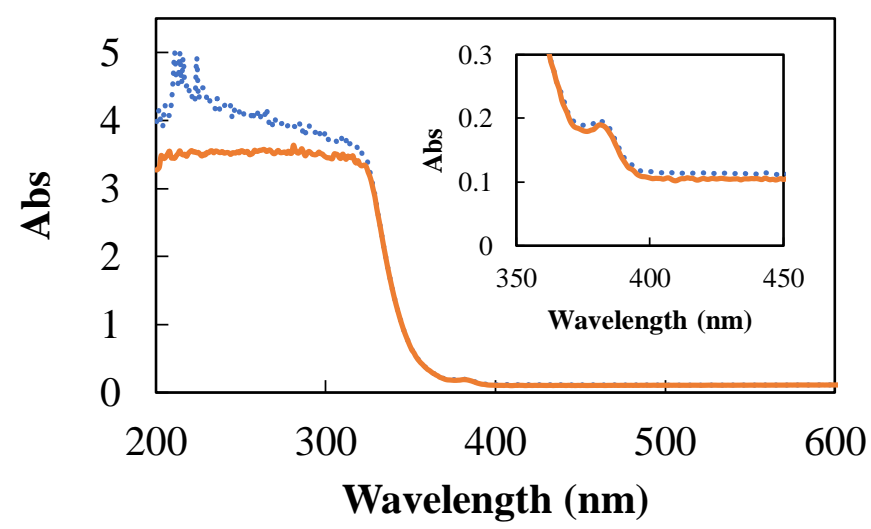

Figure 3. Absorbance spectrum of titania nanoparticles in aqueous solution $\mathrm{pH} 1$ before (--) and after (---) UV irradiation.

absorbance at $380 \mathrm{~nm}$ and the absorbance reached the detectors maximum below $355 \mathrm{~nm}$ as shown by Figure 3. Since the polymers were dissolved in THF, the nanoparticle solution was dried down and dissolved in THF. The resulting solution was orange in color (Figure 2) and had a broad absorption from 350 to $600 \mathrm{~nm}$ with a peak maximum at 425 $\mathrm{nm}$, but retained the sharp maximum at $380 \mathrm{~nm}$ as the aqueous solution shown in Figure 4. When the nanoparticle solution was mixed with the polymer solution, the orange color persisted. This orange color suggested the aggregation of aqueous nanoparticles in organic solvent. The nanoparticles showed no size dependence on DLS measurements with sizes ranging from $1 \mathrm{~nm}$ to $5 \mu \mathrm{M}$ in diameter.
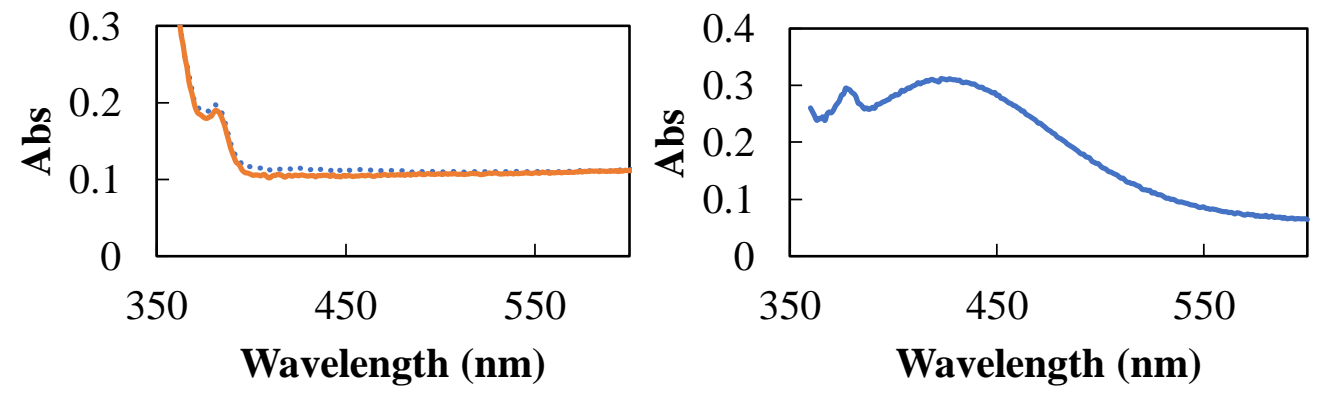

Figure 4. Absorbance spectrum of titania nanoparticles in aqueous solution $\mathrm{pH} 1$ (left) and in THF (right). 


\subsection{Polystyrene and Polymethylmethacrylate Thin Film Characterization}

A mixture of polystyrene and polymethylmethacrylate and or titania nanoparticles was dissolved in THF and spin cast onto a glass slide. The films were washed with cyclohexane and dried at $100{ }^{\circ} \mathrm{C}$ for 20 minutes. The thin films composition on a glass slide was determined from FTIR in Figure 5. The FTIR contained peaks at 2800, 2900, 3030 and $3060 \mathrm{~cm}^{-1}$ indicated that both PMMA and PS were still present in the film after washing. ${ }^{2}$ Previous literature synthesizing superhydrophobic polymers in this manner removed all PS from their films after washing with cyclohexane at $70{ }^{\circ} \mathrm{C} .{ }^{2}$ Our samples were washed at room temperature and then dried for 20 minutes which may be responsible for the discrepancy in polymer composition. Three samples shown in Figure 6 are glass slide with films composed of PMMA/PS, PMMA/PS/Titania nanoparticle mixed solutions and PMMA/PS layered with aqueous titania nanoparticles respectively. Titania nanoparticles in THF had an orange color but when the sample was washed

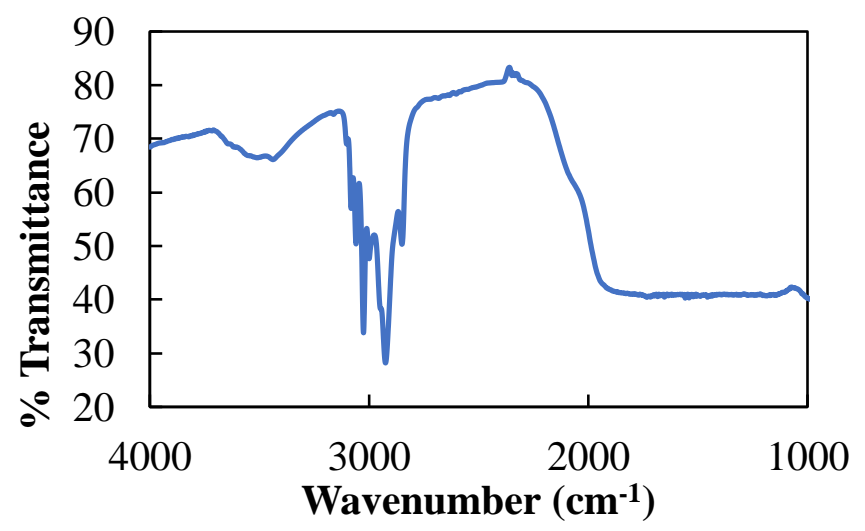

Figure 5. IR spectrum of PMMA/PS thin film washed with cyclohexane and dried at $100{ }^{\circ} \mathrm{C}$ for 20 minutes.

and dried, the color faded a produced a white film. The samples are then displayed with a water droplet. Water contact angles could not be measured for these materials at this facility. All films were hydrophobic in nature, with water sliding off the films, except for the film with a nanoparticle layer which was hydrophilic and water adhered to the surface. The SEM images of the films are displayed in Figure 8. The PMMA/PS films formed an anisotropic smooth surface with distinct grain boundaries. Grain size and shape was amorphous and varied from $2 \mu \mathrm{M}$ in diameter and below. There was no intrinsic roughness to the surface of this film, which plays an important role in wettability. However, this surface was still hydrophobic. Ti nanoparticles layered on the PMMA/PS substrate had isotropic properties with uneven coating of the polymer. The uneven layering could be due to the amorphous nature of the nanoparticles which do not have a defined size, but rather a large range of sizes. Individual nanoparticles cannot be
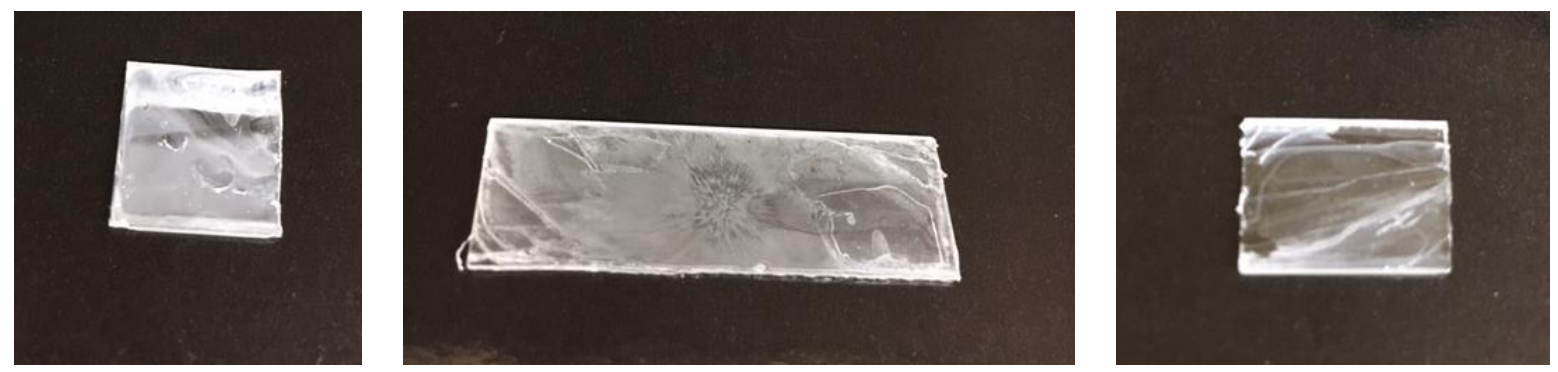

Figure 6. PMMA/PS thin film on glass substrates. PMMA/PS (left) and PMMA/PS/Titania nanoparticles mixed film (middle) and PMMA/PS layered with titania nanoparticles (right) . 

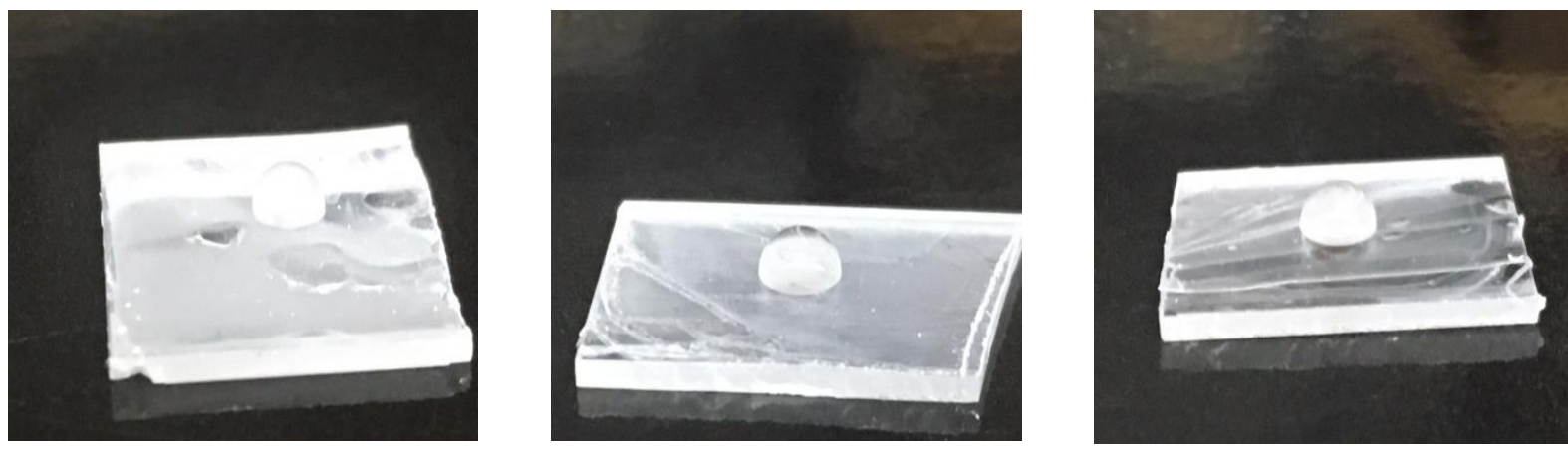

Figure 7. Hydrophobic PMMA/PS thin film on glass substrates with water droplet. PMMA/PS (left) PMMA/PS layered with titania nanoparticles (middle) and PMMA/PS/Titania nanoparticles mixed film.

seen in the SEM image but the spherical outlines are visible. The grains from the PMMA/PS substrate are no longer anisotropic in nature as the background of the image are all spheres instead of the amporphous grains as previously shown. When the titania nanoparticles were mixed with PMMA/PS solution and then spincast onto the substrate an isotropic surface formed. The nanoparticles aggregated into larger spheres from the organic solvent. Nanoparticles

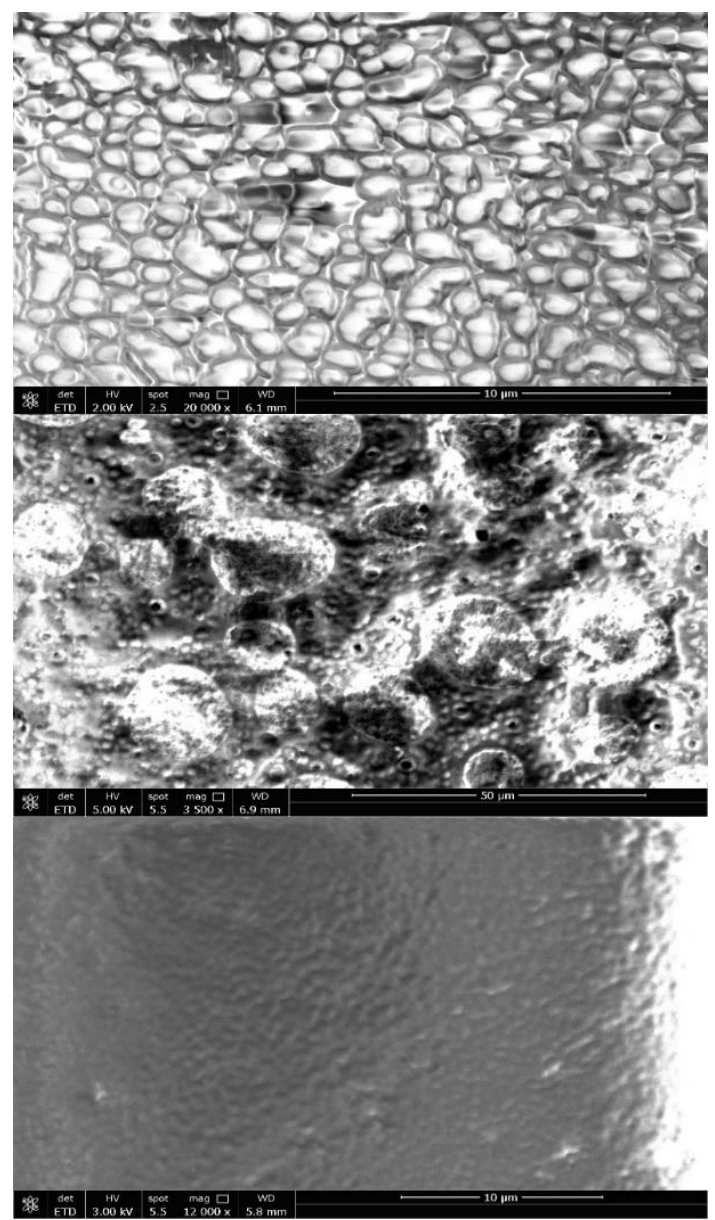

Figure 8. SEM image of PMMA/PS thin film on glass substrates. PMMA/PS at 20,000X (top) PMMA/PS/Titania nanoparticles mixed film at 3,500 X(middle) PMMA/PS layered with titania nanoparticles at 12,000X (bottom). 
were tens of nanometers to micron in size, but the aggregate which formed a sphere was $40 \mu \mathrm{M}$ in diameter. The PMMA/PS also formed spheres in response to the hydrophilic nanoparticles as the grains are no longer visible. Although for all the samples there was no distinct roughness within the film, the addition of titania nanoparticles into the film changed the surface from anisotropic to isotropic by repulsion of hydrophobic and hydrophilic counterparts. Larger magnification of these films could not be attained due to the resistivity of the substrate.

\subsection{Nanoparticle Thin Film Photolysis}

There is evidence in the literature of ultraviolet radiation responsive surfaces with titania nanoparticles. ${ }^{3}$ Our samples containing titania nanoparticles were exposed to a mercury vapor-lamp for 3 hours and the hydrophobicity was tested. SEM images in Figure 9 of layered or mixed polymer and nanoparticles solutions showed no change in appearance structurally or in color which was consistent with literature. ${ }^{3}$ The film with a layer of nanoparticles irradiated for 3



Figure 9. SEM image of PMMA/PS thin film on glass substrates. PMMA/PS/Titania nanoparticles mixed film at 2000X (top) PMMA/PS layered with titania nanoparticles at 1500X (bottom).

hours showed a clear outline of individual nanoparticles, where the unirradiated sample did not. The nanoparticle sizes ranged from tens of nanometers to micron in size. This could be due to the sample drying for a longer period of time and removing any trapped solvents. The structure of the irradiated mixed nanoparticle film remained unchanged, with large aggregates of nanoparticles and aggregates of polymer. The absorbance spectrum of irradiated nanoparticles in solution show no change in the absorbance at $380 \mathrm{~nm}$, but absorbance in the UV increased as shown in Figure. When wettability was tested, again the film with layered nanoparticles still adhered to the water droplet, where the other mixed film with nanoparticles remained hydrophobic. The water contact angle could not be measured, 
but literature has shown that even though the structure has not changed, the contact angle would.

\section{Conclusion and Future Directions}

Hydrophobic PMMA/PS blend polymer films with hydrophilic titania nanoparticles were prepared by the selective solvent method. The surface morphology of the thin films changed with the addition of nanoparticles and the method of addition. Mixing the polymers and hydrophilic titania nanoparticles in THF generated nanoparticle and polymer agglomerations on the surface of the films. Layering titania nanoparticles generated a smooth surface above the PMMA/PS base. Irradiation of these thin films did not cause a change in structure, but water contact angles which could not be measured may have been altered. These measurements will be completed at a later date. Different compositions of thin films will also be investigated, such as polystyrene substrate with polystyrene or polymethylmethacrylate nanoparticles which are hydrophobic in nature to add to the superhydrophobic polymer. Different nanoparticles that are known to be UV reactive will also be tested such as iron oxide.

\section{REFERENCES}

1. Wolfs, M., Encyclopedia of Polymer Science and Technology. John Wiley \& Sons: New York, 2002.

2. Ma, Y.; Ca, X.; Feng, X.; Ma, Y.; Zou, H., Fabrication of super-hydrophobic film from PMMA with intrinsic water contact angle below 90. Polymer, 48, 7455-7460 (2007).

3. Pant, R.; Singha, S.; Bandyopadhyay, A.; Khare, K., Investigation of static and dynamic wetting transitions of UV responsive tunable wetting surfaces. Appl. Surface Sci. 292, ,77-781, (2014).

4. Bernagozzi, I.; Torrengo, S.; Minati, L.; Ferrari, M.; Chiappini, A.; Aermellini, C.; Toniutti, L.; Lunelli, L.; Speranza, G., Synthesis and characterization of PMMA-based superhydrophobic surfaces. Colloid Poym. Sci.,290, 315-322, (2012).

5. Imran, M.; Riaz, S.; Naseem, S., Synthesis and Characterization of Titania Nanoparticles by Sol-gel Technique. Materials Today Proceed., 2 (10), 5455-5461 (2015). 